Original Paper

\title{
Evaluation of the motion of lung tumors during stereotactic body radiation therapy (SBRT) with four-dimensional computed tomography (4DCT) using real-time tumor-tracking radiotherapy system (RTRT)
}

\author{
Keiichi Harada a , Norio Katoh b,c,*, Ryusuke Suzuki ${ }^{\text {b }}$, Yoichi M. Ito ${ }^{\text {d }}$, Shinichi Shimizu c,e, \\ Rikiya Onimaru ${ }^{\text {a }}$, Tetsuya Inoue ${ }^{\mathrm{b}}$, Naoki Miyamoto ${ }^{\mathrm{b}}$, Hiroki Shirato ${ }^{\mathrm{a}, \mathrm{c}}$ \\ a Department of Radiation Medicine, Hokkaido University Graduate School of Medicine, North-15 West-7, Kita-ku, Sapporo 060-8638, Japan \\ b Department of Radiation Oncology, Hokkaido University Hospital, North-14 West-5, Kita-ku, Sapporo 060-8638, Japan \\ c Global Station for Medical Science and Engineering, Global Institution for Collaborative Research and Education, Hokkaido University Graduate School of \\ Medicine, North-15 West-7, Kita-ku, Sapporo 060-8638, Japan \\ ${ }^{\mathrm{d}}$ Department of Biostatistics, Hokkaido University Graduate School of Medicine, North-15 West-7, Kita-ku, Sapporo 060-8638, Japan \\ e Department of Radiation Oncology, Hokkaido University Graduate School of Medicine, North-15 West-7, Kita-ku, Sapporo 060-8638, Japan
}

\section{A R T I C L E I N F O}

Article history:

Received 20 August 2015

Received in revised form 9 October 2015

Accepted 23 October 2015

Available online 2 March 2016

\section{Keywords:}

Four-dimensional computed tomography

Stereotactic body radiation therapy

Real-time tumor-tracking radiotherapy

Organ motion

\begin{abstract}
A B S T R A C T
Purpose: We investigated the usefulness of four-dimensional computed tomography (4DCT) performed before stereotactic body radiation therapy (SBRT) in determining the internal margins for peripheral lung tumors.

Methods and Materials: The amplitude of the movement of a fiducial marker near a lung tumor measured using the maximum intensity projection (MIP) method in 4DCT imaging was acquired before the SBRT $\left(\right.$ Amp $_{\mathrm{CT}}$ ) and compared with the mean amplitude of the marker movement during SBRT (Ampmean) and with the maximum amplitude of the marker movement during SBRT (Ampmax $)$ using a real-time tumortracking radiotherapy (RTRT) system with 22 patients.

Results: There were no significant differences between the means of the Ampmean and the means of the Amp $_{\mathrm{CT}}$ in all directions ( $\mathrm{LR}, P=0.45$; $\mathrm{CC}, P=0.80$; AP, $P=0.65$ ). The means of the Ampmax were significantly larger than the means of the Amp $\mathrm{AT}_{\mathrm{C}}$ in all directions (LR, $\left.P<0.01 ; \mathrm{CC}, P=0.03 ; \mathrm{AP}, P<0.01\right)$. In the

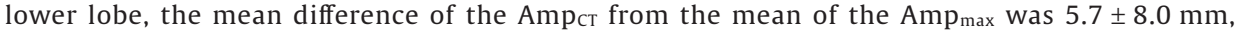
$12.5 \pm 16.7 \mathrm{~mm}$, and $6.8 \pm 8.5 \mathrm{~mm}$ in the LR, CC, and AP directions, respectively.

Conclusions: Acquiring 4DCT MIP images before the SBRT treatment is useful to establish the mean amplitude for a patient during SBRT but it underestimates the maximum amplitude during actual SBRT. Caution must be paid to determine the margin with the 4DCT especially for tumors at the lower lobe where it is of the potentially greatest benefit.

(C) 2016 Associazione Italiana di Fisica Medica. Published by Elsevier Ltd. This is an open access article under the CC BY-NC-ND license (http://creativecommons.org/licenses/by-nc-nd/4.0/).
\end{abstract}

\section{Introduction}

Stereotactic body radiation therapy (SBRT) is widely used in the therapy of localized lung malignancies with patients where the malignancy is inoperable or where patients refuse to undergo surgical resection [1-3]. It is important to reduce uncertainties in the target delineation and localization, and thereby limit the doses to the surrounding normal tissue [4-6].

\footnotetext{
* Corresponding author. Department of Radiation Oncology, Hokkaido University Hospital, North-14 West-5, Kita-ku, Sapporo 060-8638, Japan. Tel.: +81 11706 5977; fax: +81 117067876 .

E-mail address: noriwokatoh@med.hokudai.ac.jp (N. Katoh).
}

Four-dimensional computed tomography (4DCT) has been widely used to estimate the internal motion of lung cancers in SBRT. Underberg et al. have shown that 4DCT is useful to determine the internal target volume (ITV) in stereotactic body radiation therapy (SBRT) for stage I lung cancer [7]. Using 4DCT, Liu et al. have shown that the principal component of the tumor motion was in the cranialcaudal direction (CC), with only $10.8 \%$ of tumors moving $>1.0 \mathrm{~cm}$ based on the data of 4DCT [8].

The accuracy of the ITV determination based on 4DCT has been compared with other imaging modalities. Cai et al. found that ITVs based on 4DCT were comparatively smaller than those based on dynamic magnetic resonance imaging (MRI) in both phantom studies and lung tumor patient studies [9]. Purdie et al. have shown that tumor motion of the planning 4DCT scan did not match that of 
a

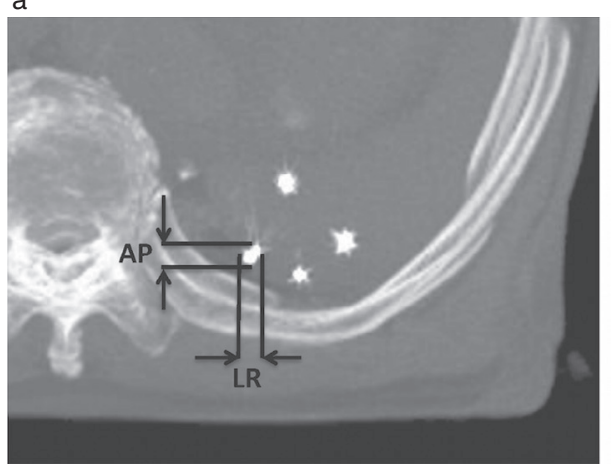

b

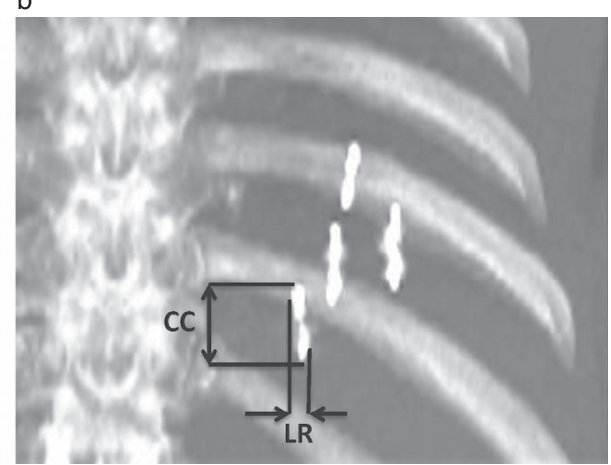

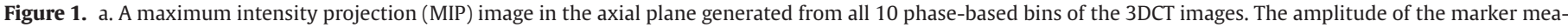

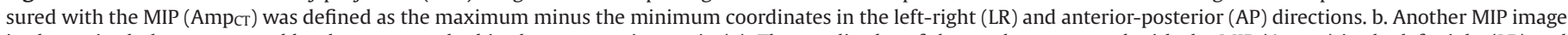

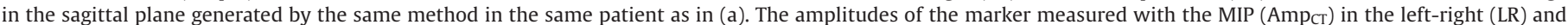
cranio-caudal (CC) directions are shown.

cone-beam CT on the treatment unit at the start of treatment in 2 of 12 patients [10]. They suggested that ITV based on 4DCT could underestimate the tumor motion and not be suitable for patients receiving SBRT. However, there is little information about the accuracy of the motion estimated from 4DCT in comparison with the actual internal motion of tumors during SBRT.

The internal motion of tumors has been shown to vary in the same patient by analysis of internal fiducial marker movements near the tumor using a real-time tumor-tracking radiotherapy (RTRT) system [11]. The purpose of this study is to evaluate the accuracy of the amplitude of internal fiducial markers in the lung by comparing 4DCT data with RTRT log data obtained during SBRT in the same patient. We have not compared displacement of the tumor location between treatments, which would be relevant to the so called 'inter-fractional motion'. We have compared the amplitude of the tumor motion during the treatment, which is more relevant to the so called 'intra-fractional motion'.

\section{Methods and materials}

\section{Patients}

We obtained approval from the research ethics committee of Hokkaido University Hospital for this retrospective study (No. 0120395). From May 2011 to October 2013, 22 patients with peripheral lung tumors, who underwent 4DCT and SBRT with a RTRT system, were included in this study. The median age was 77.5 years (range 63-89). Tumor locations were: 11 upper-middle lobes and 11 lower lobes (Table S1). As previously described, in a RTRT system, the threedimensional (3D) coordinates of a fiducial marker which has been implanted near the lung tumor were recorded every 0.033 seconds during the SBRT using two sets of fluoroscopes [12]. The outcome of the SBRT using RTRT has been reported elsewhere [13]. In our institution, we used RTRT for the lung SBRT using the RTRT system. The RTRT is basically a gated radiotherapy where the therapeutic beam is delivered only when the internal fiducial marker near the tumor is within a $\pm 2-\mathrm{mm}$ of the planned position at the end of expiration. This is the reason why we used breath-hold CT images acquired at the end of expiration for the determination of CTV and for dose calculations. Five mm was added to the CTV to make the PTV. The 4DCT image was used for research purposes in this study. The prescription was $40 \mathrm{~Gy}$ in 4 fractions to the $95 \%$ of PTV in principle.

\section{Measurement of amplitudes using 4DCT}

Fiducial markers with a diameter of $2 \mathrm{~mm}$ were implanted through endoscopy before the 4DCT. For each lung tumor, 3-4 markers were implanted near the tumor. No training or visual monitoring of breath was used during the 4DCT and RTRT. Patients were breathing freely and naturally throughout the 4DCT and SBRT procedures in this study. The marker nearest the tumor center is used for the RTRT. The 4DCT scan was performed on a 16-slice CT scanner (Optima CT580 W; GE Healthcare, Waukesha, WI) within one week before the SBRT, with transaxial images acquired during free breathing in the cine mode. The scan parameters were: $1.0 \mathrm{~s}$ gantry rotation, $0.5 \mathrm{~s}$ cine interval, $20 \mathrm{~mm}$ beam collimation, and $2.5-\mathrm{mm}$ slice thickness. The datasets at each table position were acquired for at least the duration of one respiratory cycle of the patient. During the CT scan, the respiratory signals of each patient were recorded and monitored using a Varian Real-time Position Management (RPM) system (Varian Medical Systems, Palo Alto, CA): a box with two infrared reflective markers was placed on the upper abdomen of the patient and its movement was captured by an infrared camera. The raw 4DCT images and the corresponding respiratory signal data were transferred to an Advantage Workstation 4.5 (GE Healthcare, Waukesha, WI) to sort the 4DCT images into 10 respiratory phase-based bins of 3DCT images. Maximum intensity projection (MIP) images were automatically generated from all 10 phase-based bins of the 3DCT images using the Advantage 4D software (GE Healthcare, Waukesha, WI).

We used the MIP images from the 4DCT (4DCT MIP) for the measurements of the amplitude of the fiducial marker that was tracked by the RTRT system during the SBRT. The window width was set to $2000 \mathrm{HU}$ and the window level as $150 \mathrm{HU}$, and defined a highdensity area over $1000 \mathrm{HU}$ as the trajectory of the marker. We selected areas of more than $1000 \mathrm{HU}$ as the trajectory of the marker to distinguish metallic fiducial markers from bone or calcification. The amplitude of the marker measured with the 4DCT MIP (Amp $\mathrm{AT}_{\mathrm{CT}}$ ) was defined as the maximum coordinates minus the minimum coordinates in the left-right (LR), cranial-caudal (CC), and anteriorposterior (AP) direction coordinates, respectively (Fig. 1). The 3D scalar amplitude (3DSA) was defined as the $\mathrm{Amp}_{\mathrm{CT}}$ distance in $3 \mathrm{D}$ coordinates and calculated as $\mathrm{DSA}=\left(\mathrm{LR}^{2}+\mathrm{CC}^{2}+\mathrm{AP}^{2}\right)^{1 / 2}$.

\section{Measurement of amplitudes during SBRT using RTRT log data}

In the RTRT system, log files are created continuously during the delivery of the therapeutic beam for each port unless there is a 


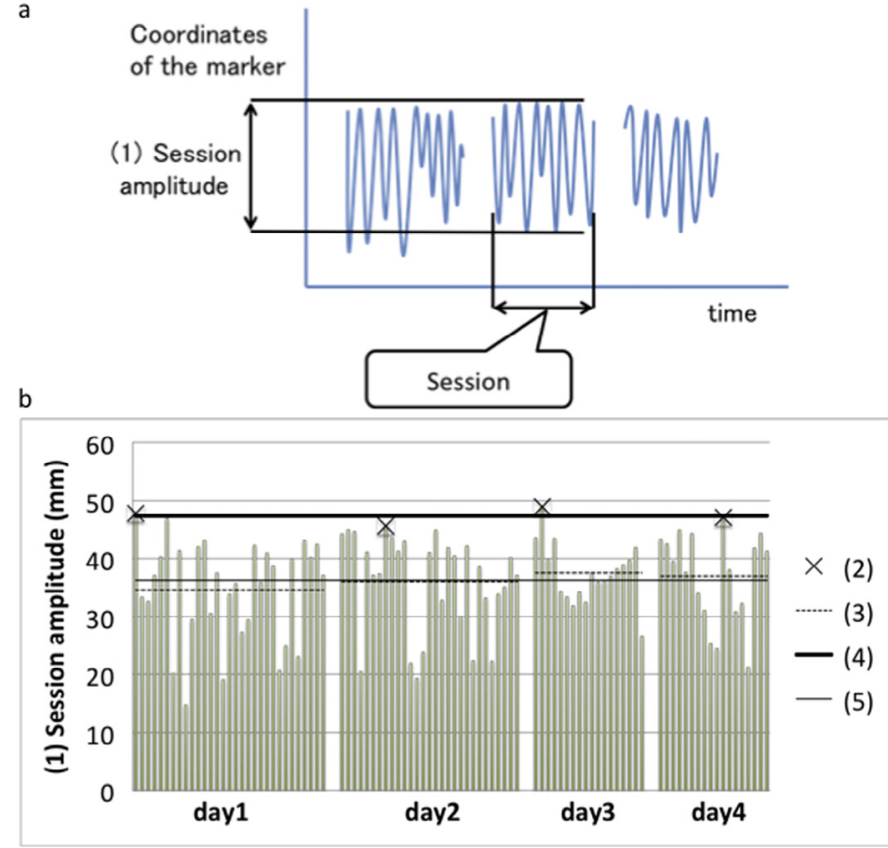

Figure 2. a. Definition of the amplitude of the marker determined in RTRT. Each continuous recording is one session. In each session, the maximum minus the minimum is the difference in the LR, CC, and AP directions. b. Example of the RTRT amplitude of one patient. (1) Session amplitude, (2) the maximum amplitude of the day, (3) the mean amplitude of the day, (4) the maximum amplitude of the patient during SBRT (Amp $\left.p_{\max }\right),(5)$ the mean amplitude of the patient during SBRT (Ampmean).

baseline shift or recognition of the marker is insufficient; in such situations, tracking of the marker stopped and the recording of the $\log$ file is discontinued. A continuous recording is termed as one 'session' here and thus each session is recorded in one log file (Fig. 2a).

We measured several types of amplitudes for each patient in this study in the LR, CC, and AP directions: (1) session amplitude, the maximum amplitude in each session; (2) the maximum amplitude of the day, the maximum session amplitude on the day; (3) the mean amplitude of the day, the mean of session amplitudes of the day; (4) the maximum amplitude of the patient during SBRT $\left(A m p_{\max }\right)$, the mean of the maximum amplitudes of one day for the whole of the treatment; (5) the mean amplitude of the patient during SBRT (Amp mean), the average of the mean amplitudes of the day during the whole of the treatment (Fig. 2b).

\section{Statistical analysis}

The JMP 9 (SAS, Cary, NC) software was used for the statistical analysis. The $A_{\mathrm{mp}}$ was compared with the mean of the Ampmean and $A_{m p} p_{\max }$ using the Wilcoxon test. The mean difference of the $A_{\mathrm{mp}}$ from the mean of the Ampmean and Amp $\mathrm{Amax}_{\text {was }}$ compared for the upper-middle and the lower lobes also using the Wilcoxon test. The statistical relationships between several clinical characteristics and the difference in the Amp $\mathrm{Am}_{\mathrm{C}}$ versus the $\mathrm{Amp}_{\text {mean }}$ and the $\mathrm{Amp}_{\max }$ were investigated using analysis of variance (ANOVA). The restricted maximum likelihood (REML) method in linear random effects model was used to estimate the variance components. As the clinical characteristics in the analysis, the age, the forced expiratory volume in 1 second (FEV1.0), lobe, and the 3DSA were used. In clinical practice, emphysematous and fibrotic changes in lung tissue are more common in old age. Also FEV1.0 is often changed in the diseased lung tissue. Therefore, we have included these parameters in the analysis. For each factor, the median value was used to divide the whole of the obtained data into two groups: ages 77 or younger vs. 78 or older, FEV1.0 $<1.8 \mathrm{~L}$ vs. $\geq 1.8 \mathrm{~L}$ or larger, lobe upper-middle vs. lower and 3DSA $<10 \mathrm{~mm}$ vs. $\geq 10 \mathrm{~mm}$ or larger. The means of the differences in the amplitudes were compared between groups using the Wilcoxon test. A $P$ value $<0.05$ was considered to show statistical significance.

\section{Results}

The mean period between the dates of the 4DCT scan and the start of the SBRT was 5.6 days (range 3-6). The duration for acquisition of 4DCT images ranged from 87.8 to 139.5 seconds (mean 104.6). In 96 treatments with the 22 patients, the length of the logdata of the RTRT system for one treatment was from 395 to 1924.3 seconds (mean 1025.5). The duration of the SBRT was about 10 times longer than the 4DCT. The average length of session of RTRT log data was 92.4 seconds (range: 49.6-174.2). There were 11.3 sessions per fraction of SBRT.

\section{Measurement of amplitudes using 4DCT}

The mean of the $A \mathrm{mp}_{\mathrm{cT}}$ in the 22 patients was $3.6 \pm 1.3 \mathrm{~mm}$ (range 1.9-6.4), $9.4 \pm 8.1 \mathrm{~mm}(1.9-36.1)$, and $5.5 \pm 1.9 \mathrm{~mm}$ (3.4-10.4) in the LR, CC, and AP directions, respectively. The median 3DSA was $9.8 \mathrm{~mm}$ (range 4.6-37.7) (Tables S1 and S2).

\section{Analysis based on the mean amplitude of each patient during SBRT}

The mean of the Amp mean was $4.2 \pm 2.8 \mathrm{~mm}$ (0.9-13), $11.4 \pm 11.6 \mathrm{~mm}(1.1-38.0)$, and $5.7 \pm 3.6 \mathrm{~mm}(1.5-15.7)$ in the $\mathrm{LR}, \mathrm{CC}$, and AP directions, respectively (Table S2). There were no significant differences between the mean of the Amp mean and the mean of the $A \mathrm{Pp}_{\mathrm{CT}}$ in any direction (LR, $P=0.45 ; \mathrm{CC}, P=0.80$; and AP, $P=0.65$ ) (Fig. 3a).

In the upper-middle lobe, the mean difference from the amplitude measured with the 4DCT to the mean of the $\mathrm{Amp}_{\text {mean }}$ in the 22 patients was $-0.2 \pm 0.8 \mathrm{~mm},-1.2 \pm 2.7 \mathrm{~mm}$, and $-0.9 \pm 1.1 \mathrm{~mm}$ in the $\mathrm{LR}, \mathrm{CC}$, and AP directions, respectively. In the lower lobe, it was $1.5 \pm 3.5 \mathrm{~mm}, 5.2 \pm 11.7 \mathrm{~mm}$, and $1.2 \pm 3.8 \mathrm{~mm}$ in the LR, CC, and AP directions, respectively. There were no statistically significant differences between the differences in the lower lobe and in the uppermiddle lobe in any of the directions ( $L R, P=0.15$; CC, $P=0.19$; and AP, $P=0.26$ ).

\section{Analysis based on the maximum amplitude of a patient during SBRT}

The mean of the Amp max $_{\text {mal }}$ of all patients was $7.0 \pm 6.0 \mathrm{~mm}(1.7-$ 28.8), $15.9 \pm 16.4 \mathrm{~mm}(2.0-52.3)$, and $9.5 \pm 7.5 \mathrm{~mm}(2.4-34.8)$ in the LR, CC, and AP directions, respectively (Table S2). The mean of the $A m p_{\max }$ was statistically significantly larger than the $A m p_{\mathrm{CT}}$ in all three directions (LR, $P<0.01 ; C C, P=0.03$; and AP, $P<0.01$ ) (Fig. $3 b$ ).

In the upper-middle lobe, the mean difference between the Amp $\mathrm{AT}_{\mathrm{CT}}$ and the mean of the $A \mathrm{mp}_{\max }$ was $1.3 \pm 1.1 \mathrm{~mm}, 0.6 \pm 3.1 \mathrm{~mm}$, and $1.1 \pm 1.7 \mathrm{~mm}$ in the LR, CC, and AP directions. In the lower lobe, it was $5.7 \pm 8.0 \mathrm{~mm}, 12.5 \pm 16.7 \mathrm{~mm}$, and $6.8 \pm 8.5 \mathrm{~mm}$ in the $\mathrm{LR}, \mathrm{CC}$, and AP directions. The differences in the lower lobe were larger than those in the upper-middle lobe in the CC and AP directions (LR, $P=0.15$; CC, $P<0.05$; and AP, $P=0.21$ ) (Fig. 4). Figure 5 illustrates the position of the markers and the differences between the means of the Amp max $_{\text {and }}$ Amp

The mean and standard deviations of the difference between the $A m p_{c T}$ and the $A m p_{\max }$ for the selected clinical characteristics (age, FEV1.0, lobe, and 3DSA) are shown in Table 1. The difference was significantly larger in the lower lobe than in the upper-middle lobe in the CC (Upper-middle $0.6 \pm 3.1$, Lower $12.5 \pm 16.7, P<0.05$ ). The difference was also larger for those with 3DSA $10 \mathrm{~mm}$ or larger than 


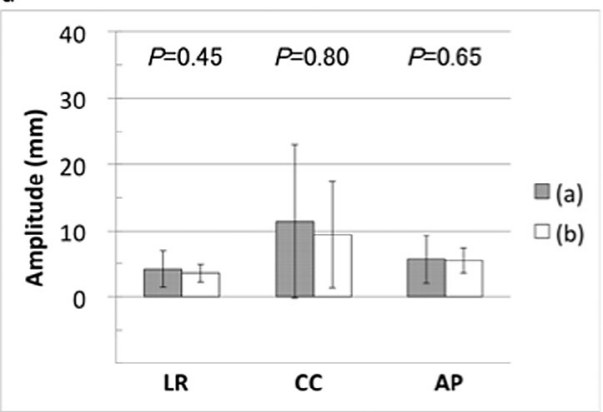

b

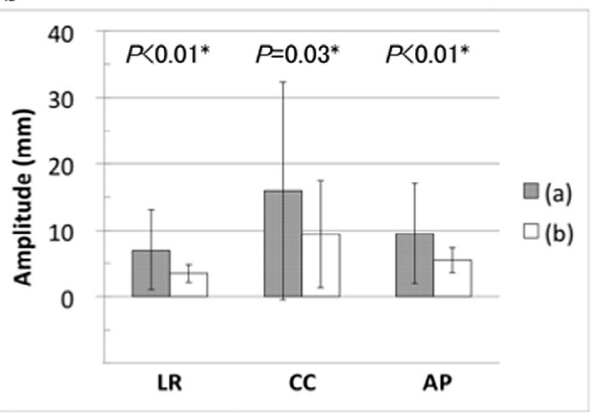

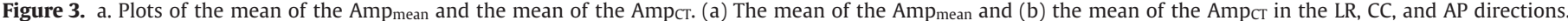
b. Plots of the mean of the Amp $\max$ and the mean of the Ampcr. (a) The mean of the Amp $\max$ and (b) the mean of the Amp in the LR, CC, and AP directions.

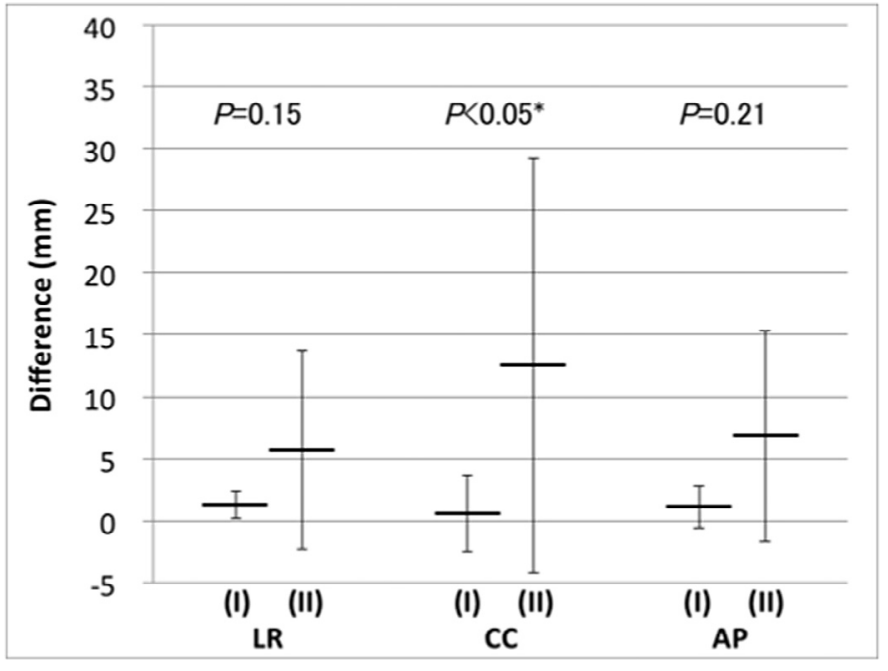

Figure 4. The mean differences of the Ampcr from the Ampmax. (I) In the uppermiddle lobe and (II) in the lower lobe for the LR, CC, and AP directions. those with 3DSA less than $10 \mathrm{~mm}$ in the LR direction $(<10 \mathrm{~mm}$ $0.9 \pm 1.7,10 \mathrm{~mm} 6.0 \pm 1.7, P=0.01)$. No other statistical differences were observed.

Relationships between the several clinical characteristics and the mean difference between the Amp $\mathrm{p}_{\mathrm{cT}}$ and the mean of the Amp $\mathrm{p}_{\max }$ were investigated using ANOVA. Estimated percentages of total variance components using the REML method were determined as shown in Table 2. For age, FEV1.0, lobe, and 3DSA, it was $0.0 \%, 4.4 \%$, $5.1 \%$, and $24.0 \%$, respectively, in the LR direction; $0.0 \%, 1.1 \%, 29.7 \%$, and $0.0 \%$ in the CC direction; and $0.0 \%, 0.0 \%, 21.3 \%$, and $5.5 \%$ in the AP direction. These percentages suggest that the lobe and the 3DSA contribute to the mean difference in the LR, CC, and AP directions (Table 2). However, the residual component other than these clinical characteristics in the variance is still large in all three directions.

\section{Discussion}

As a simple approach to generate individualized ITVs from 4DCT, Ezhil et al. have investigated the accuracy of MIP-based ITV compared with the method of contouring on all 10 phases [14]. Here it was found that the MIP-based ITV underestimated the volume
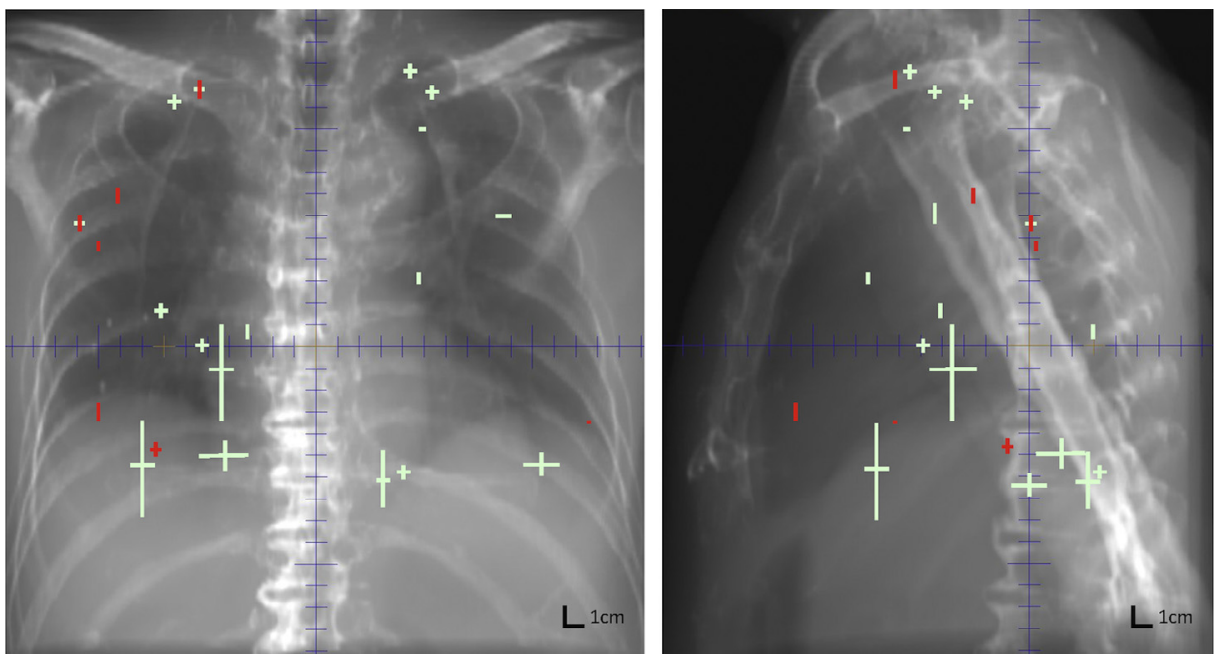

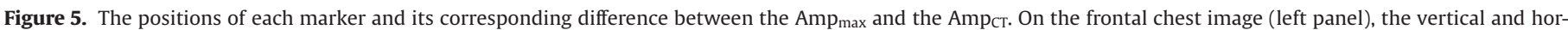

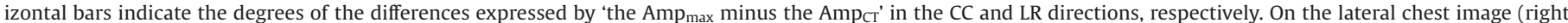

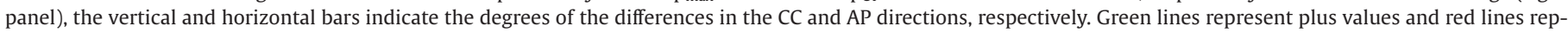
resent minus values of the differences. (For interpretation of the references to color in this figure legend, the reader is referred to the web version of this article.) 
Table 1

Difference of the Ampct from the mean of the Ampmax (Mean \pm Standard Deviation).

\begin{tabular}{|c|c|c|c|c|c|c|c|c|c|c|c|c|}
\hline & \multicolumn{3}{|l|}{ Age } & \multicolumn{3}{|l|}{ FEV1.0 } & \multicolumn{3}{|l|}{ Lobe } & \multicolumn{3}{|l|}{ 3DSA } \\
\hline & $\begin{array}{l}<77 \\
(n=11)\end{array}$ & $\begin{array}{l}78 \leq \\
(n=11)\end{array}$ & $P$ & $\begin{array}{l}<1.8 \mathrm{~L} \\
(\mathrm{n}=11)\end{array}$ & $\begin{array}{l}1.8 \mathrm{~L} \leq \\
(\mathrm{n}=11)\end{array}$ & $P$ & $\begin{array}{l}\text { Upper-middle } \\
(\mathrm{n}=11)\end{array}$ & $\begin{array}{l}\text { Lower } \\
(n=11)\end{array}$ & $P$ & $\begin{array}{l}<10 \mathrm{~mm} \\
(\mathrm{n}=11)\end{array}$ & $\begin{array}{l}10 \mathrm{~mm} \leq \\
(\mathrm{n}=11)\end{array}$ & $P$ \\
\hline $\mathrm{LR}(\mathrm{mm})$ & $3.1 \pm 3.7$ & $3.9 \pm 7.9$ & 0.51 & $4.6 \pm 8.2$ & $2.4 \pm 2.4$ & 0.74 & $1.3 \pm 1.1$ & $5.7 \pm 8.0$ & 0.15 & $0.9 \pm 1.7$ & $6.0 \pm 1.7$ & $0.010^{*}$ \\
\hline $\mathrm{CC}(\mathrm{mm})$ & $5.0 \pm 8.0$ & $8.1 \pm 17.3$ & 0.43 & $5.2 \pm 12.1$ & $7.9 \pm 14.8$ & 0.65 & $0.6 \pm 3.1$ & $12.5 \pm 16.7$ & $0.049^{*}$ & $4.0 \pm 11.9$ & $9.1 \pm 14.6$ & 0.17 \\
\hline $\mathrm{AP}(\mathrm{mm})$ & $3.7 \pm 4.8$ & $4.3 \pm 8.3$ & 0.36 & $5.4 \pm 8.7$ & $2.5 \pm 3.5$ & 1.0 & $1.1 \pm 1.7$ & $6.8 \pm 8.5$ & 0.21 & $1.7 \pm 4.6$ & $6.2 \pm 7.8$ & 0.10 \\
\hline
\end{tabular}

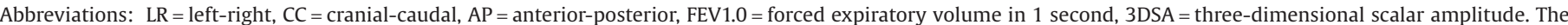
asterisk in Table 1 shows the statistical significance $(P<0.05)$.

in stage I and stage III NSCLC patients. It was further pointed out that the MIP images may not fully display mobile structures if the adjacent structures have similar densities, which is the case for lesions located near the mediastinum, diaphragm, liver, and chest wall. Mancosu et al. have proposed a semiautomatic technique for defining the internal margins of lung tumors close to the liver cupola by $4 \mathrm{DCT}$ to overcome measurement errors due to the limitations of MIP images [15]. In the present study, there are possible measurement errors by metal marker artifacts on the 4DCT MIP images. However, since the CT image number is much higher than that of the surrounding tissue, we considered that any ambiguity in the motion measurements was less than in the measurements of the tumor mass which is composed of soft tissue and would present the possibility of partial volume effects at the edge of the tumor.

The present study showed that the mean of the Ampmean was not statistically different from the mean of the $\mathrm{Amp}_{\mathrm{CT}}$ in all three of the LP, CC, and AP directions. This allows the conclusion that, as long as the object is to determine the Ampmean it is sufficient and acceptable to use the Ampст.

At the same time, the present study also showed that the mean

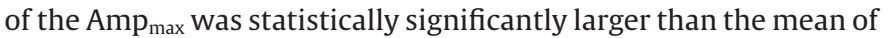
the Amp $\mathrm{AT}_{\mathrm{C}}$ in all of the LP, CC, and AP directions. This is consistent with previous suggestions of possible underestimation of the maximum amplitudes measured with 4DCT MIP images [9]. The reason why $A_{\mathrm{mp}}$ is underestimated may be because the reconstructed 4DCT MIP images are vulnerable to variations in respiratory motion and to differences between internal respiratory motion $[16,17]$ as well as to the motion of the skin surface marker used in 4DCT.

The longer time period for the delivery of SBRT compared to the time needed for the 4DCT may also be a cause. Baseline drift of the respiratory motion and changes in the depth of respiration may occur during the longer period of the SBRT [18]. Remaining and new challenges of radiotherapy of $4 \mathrm{D}$ imaging have been reported previously [19].

The mean difference between the Amp $\mathrm{p}_{\mathrm{cr}}$ and the mean of the $\mathrm{Amp}_{\max }$ was larger in the lower lobe. This may be because of an inaccurate reconstruction due to higher speed of motion of the tumor in the lower lobe [11]. It is reasonable to expect that the smaller motion in the upper and middle lobes resulted in the smaller differences in the amplitudes of the upper and middle lobes. These results do not disagree with previous results of differences in the three-dimensional trajectory of skin surface and internal fiducial markers either [20].

In general, previous reports have suggested that 4DCT can be expected to be useful especially for the lower lobe where the tumor motion is large. However, the present study showed that the difference is significantly larger in the lower lobe. This result stresses that it is not safe to reduce the internal margin for tumors in the lower lobe by using an ITV solely based on the 4DCT MIP. The magnitude of the difference reached $12.5 \pm 16.7 \mathrm{~mm}$ in the CC direction, leading us to suggest that it cannot be recommended to use 4DCT to estimate the internal margin for tumors in the lower lobe in general. For tumors in the upper and middle lobes, Onodera et al. have reported that an insignificant proportion of these tumors have large amplitudes in patients with poor pulmonary function and in patients who have a history of surgical operations of the thorax [21]. Based on these results, 4DCT may be considered adequate to estimate the internal margins for tumors at the upper and middle lobes in patients with normal pulmonary function.

In dynamic tracking of radiation therapy with real-time monitoring, the margins added to the clinical target volume (CTV) can in principle be kept smaller than those determined for SBRT in freebreathing [22-24]. However, our results suggest that extreme caution must be paid not to miss-estimate the CTV when the external skin surface or surrogate signals are used during the dynamic tracking of a tumor based on the 4DCT data. Similarly, the most careful attention should be paid to passive scattering particle therapy and even more caution shown with intensity modulated radiotherapy and spot scanning particle therapy where interplay effects of beam and organ motion may deteriorate the dose distribution further.

It could be possible to improve the accuracy of the 4DCT by increasing the time period where images are taken but this would result in additional X-ray exposure. Also the difference in the respiratory pattern at the treatment planning and at the actual treatment will not be overcome by increasing the time for the 4DCT. This study found that 3DSA and the position of the lobe contributed to the variation in the difference between treatment planning and the actual treatment patterns. However, the residual

Table 2

Variance components for the mean of the Ampcr around the mean of the Ampmax.

\begin{tabular}{|c|c|c|c|c|c|c|c|c|c|}
\hline & \multicolumn{3}{|l|}{ LR } & \multicolumn{3}{|l|}{$\mathrm{CC}$} & \multicolumn{3}{|l|}{$\mathrm{AP}$} \\
\hline & Variance $\left(\mathrm{mm}^{2}\right)$ & $95 \% \mathrm{CI}\left(\mathrm{mm}^{2}\right)$ & $\% *$ & Variance $\left(\mathrm{mm}^{2}\right)$ & $95 \% \mathrm{CI}\left(\mathrm{mm}^{2}\right)$ & $\% *$ & Variance $\left(\mathrm{mm}^{2}\right)$ & $95 \% \mathrm{CI}\left(\mathrm{mm}^{2}\right)$ & $\% *$ \\
\hline Age & 0 & 0 & 0.0 & 0 & 0 & 0.0 & 0 & 0 & 0.0 \\
\hline FEV1.0 & 1.9 & $0.2-8.7$ & 4.4 & 2.3 & $0.6-4.0$ & 1.1 & 0 & 0 & 0.0 \\
\hline Lobe & 2.2 & $0.2-3.0$ & 5.1 & 61.4 & $9.9-3,106,996.7$ & 29.7 & 10.5 & $1.6-2,857,152.6$ & 21.3 \\
\hline 3DSA & 10.3 & $1.5-8,265,330.5$ & 24.0 & 0 & 0 & 0.0 & 2.7 & $0.3-1.8$ & 5.5 \\
\hline Residual & 28.5 & $16.3-61.9$ & 66.5 & 143.4 & $82.3-310.4$ & 69.2 & 36.2 & $21.0-76.6$ & 73.2 \\
\hline Total & 42.9 & & 100.0 & 207.1 & & 100.0 & 49.4 & & 100.0 \\
\hline
\end{tabular}

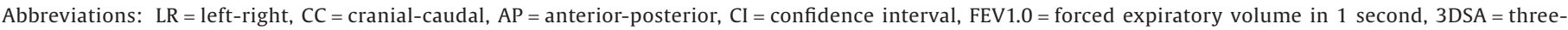
dimensional scalar amplitude.

* Contribution of clinical characteristics to the total variance was shown as the percentage. 
component of variance was too large to be able to confidently predict the difference by using the clinical characteristics identified before the treatment. The large residual component is probably reflecting randomness in the respiratory motion and would be difficult to reduce by adding further clinical characteristics in the analysis.

Gated radiotherapy using the RTRT system has been suggested to be useful to reduce the amount of residual error both for lung as well as for abdominal tumors because of the real-time monitoring of the tumor position [25]. The safety and efficacy of RTRT for treatment of stage I NSCLC has also been reported [13]. However, caution must also be paid to possible dislocation of the fiducial marker during the actual treatment (making its position different from that at treatment planning) adding additional differences between the motion of a tumor and the motion of the fiducial marker near the tumor [26,27]. Jang et al. reported the importance of setting an appropriate gaiting window regarding tumor characteristics as necessary in gated SBRT in general for lung cancer [28].

The limitations of the present study relate to these problems of gated SBRT using an RTRT system and fiducial markers. The relationship between the tumor motion and that of the fiducial marker motion may change during the treatment. Ueki et al. reported that the root mean squares of the standard deviations for each phase were $0.6,0.9$, and $1.5 \mathrm{~mm}$ in the right-left, anterior-posterior, and superior-inferior directions, respectively [29]. Therefore, since this study is dealing with the discrepancies between the 4DCT and RTRT for fiducial markers, the results should be interpreted with caution. When applying the present results for general purposes, the possible discrepancy between the tumor itself and the fiducial markers must be considered. Further investigation will be required to more accurately detect real-time three-dimensional motion of the CTV during the delivery of the irradiation.

\section{Conclusion}

The present study found that determining the amplitude measured with 4DCT MIP before SBRT treatment is adequate to know the mean amplitude for a patient prior to SBRT. However, the study also found that amplitudes measured with 4DCT MIP underestimate the maximum amplitude of tumors at the lower lobe. This stresses that great care must be paid to determine the margin of organ motion accurately with 4DCT especially for tumors at the lower lobe when the benefits of the SBRT here are especially critical.

\section{Conflict of interest}

The Corresponding Author (N.K.) has received grants from a Grant-in-Aid for Young Scientists (B) from the Ministry of Education, Culture, Sports, Science and Technology of the Japanese Government (No. 24791260) during the conduct of the study.

R.S. has received grants from a Grant-in-Aid for Young Scientists (B) from the Ministry of Education, Culture, Sports, Science and Technology of the Japanese Government (No. 24791264) during the conduct of the study.

Y.M.I. has received grants from Nihon Medi-Physics Corporation, personal fees from Japan Tobacco Inc. and personal fees from Ono Pharmaceutical Co., Ltd. outside the submitted work.

R.O. has received personal fees from Janssen Pharmaceutical K.K. and personal fees from Shimadzu Corporation outside the submitted work.

H.S. has received grants from the Government during the conduct of the study; grants from Hitachi, Ltd., grants and personal fees from Mitsubishi Heavy Industries, Ltd., grants and personal fees from Shimadzu Corporation, grants and personal fees from Varian Medical Systems, Inc., and personal fees from Olympus Corporation outside the submitted work. In addition, H.S. holds a patent US 6,307,914 with royalties paid.

\section{Acknowledgements}

This study has been presented in 55th annual meeting of American Society of Radiation Oncology, September 22-25, 2013, Atlanta, GA. Further, this study was supported by grants from a Grant-inAid for Young Scientists (B) from the Ministry of Education, Culture, Sports, Science and Technology of the Japanese Government (Nos. 24791260 and 24791264).

\section{Appendix Supplementary material}

Supplementary data to this article can be found online at doi:10.1016/j.ejmp.2015.10.093.

\section{References}

[1] Onishi H, Shirato H, Nagata Y, Hiraoka M, Fujino M, Gomi K, et al. Stereotactic body radiotherapy (SBRT) for operable stage I non-small-cell lung cancer: can SBRT be comparable to surgery? Int J Radiat Oncol Biol Phys 2011;81:1352-8.

[2] Onishi H, Shirato H, Nagata Y, Hiraoka M, Fujino M, Gomi K, et al. Hypofractionated stereotactic radiotherapy (HypoFXSRT) for stage I non-small cell lung cancer: updated results of 257 patients in a Japanese multi-institutional study. J Thorac Oncol 2007;2:S94-100.

[3] Onimaru R, Fujino M, Yamazaki K, Onodera Y, Taguchi H, Katoh N, et al. Steep dose-response relationship for stage I non-small-cell lung cancer using hypofractionated high-dose irradiation by real-time tumor-tracking radiotherapy. Int J Radiat Oncol Biol Phys 2008;70:374-81.

[4] Nagata Y, Hiraoka M, Mizowaki T, Narita Y, Matsuo Y, Norihisa Y, et al. Survey of stereotactic body radiation therapy in Japan by the Japan 3-D Conformal External Beam Radiotherapy Group. Int J Radiat Oncol Biol Phys 2009;75:343-7.

[5] Matsuo Y, Onishi H, Nakagawa K, Nakamura M, Ariji T, Kumazaki Y, et al. Guidelines for respiratory motion management in radiation therapy. J Radiat Res 2013;54:561-8.

[6] Onimaru R, Shirato H, Shimizu S, Kitamura K, Xu B, Fukumoto S, et al. Tolerance of organs at risk in small-volume, hypofractionated, image-guided radiotherapy for primary and metastatic lung cancers. Int J Radiat Oncol Biol Phys 2003;56:126-35.

[7] Underberg RW, Lagerwaard FJ, Cuijpers JP, Slotman BJ, van Sornsen de Koste JR, Senan S. Four-dimensional CT scans for treatment planning in stereotactic radiotherapy for stage I lung cancer. Int J Radiat Oncol Biol Phys 2004;60:128390.

[8] Liu HH, Balter P, Tutt T, Choi B, Zhang J, Wang C, et al. Assessing respirationinduced tumor motion and internal target volume using four-dimensional computed tomography for radiotherapy of lung cancer. Int J Radiat Oncol Biol Phys 2007;68:531-40.

[9] Cai J, Read PW, Baisden JM, Larner JM, Benedict SH, Sheng K. Estimation of error in maximal intensity projection-based internal target volume of lung tumors: a simulation and comparison study using dynamic magnetic resonance imaging. Int J Radiat Oncol Biol Phys 2007;69:895-902.

[10] Purdie TG, Moseley DJ, Bissonnette JP, Sharpe MB, Franks K, Bezjak A, et al. Respiration correlated cone-beam computed tomography and 4DCT for evaluating target motion in Stereotactic Lung Radiation Therapy. Acta Oncol 2006;45:915-22.

[11] Shirato H, Suzuki K, Sharp GC, Fujita K, Onimaru R, Fujino M, et al. Speed and amplitude of lung tumor motion precisely detected in four-dimensional setup and in real-time tumor-tracking radiotherapy. Int J Radiat Oncol Biol Phys 2006;64:1229-36.

[12] Shirato H, Onimaru R, Ishikawa M, Kaneko J, Takeshima T, Mochizuki K, et al. Real-time 4-D radiotherapy for lung cancer. Cancer Sci 2012;103:1-6.

[13] Inoue T, Katoh N, Onimaru R, Shimizu S, Tsuchiya K, Suzuki R, et al. Stereotactic body radiotherapy using gated radiotherapy with real-time tumor-tracking for stage I non-small cell lung cancer. Radiat Oncol 2013;8:69.

[14] Ezhil M, Vedam S, Balter P, Choi B, Mirkovic D, Starkschall G, et al. Determination of patient-specific internal gross tumor volumes for lung cancer using fourdimensional computed tomography. Radiat Oncol 2009;4:4.

[15] Mancosu P, Sghedoni R, Bettinardi V, Aquilina MA, Navarria P, Cattaneo GM et al. Semiautomatic technique for defining the internal gross tumor volume of lung tumors close to liver/spleen cupola by 4D-CT. Med Phys 2010;37:4572-6.

[16] Bouilhol G, Ayadi M, Rit S, Thengumpallil S, Schaerer J, Vandemeulebroucke J et al. Is abdominal compression useful in lung stereotactic body radiation therapy? A 4DCT and dosimetric lobe-dependent study. Phys Med 2013;29:333-40.

[17] Aznar MC, Persson GF, Kofoed IM, Nygaard DE, Korreman SS. Irregular breathing during 4DCT scanning of lung cancer patients: is the midventilation approach robust? Phys Med 2014;30:69-75.

[18] Seppenwoolde Y, Shirato H, Kitamura K, Shimizu S, van Herk M, Lebesque JV, et al. Precise and real-time measurement of 3D tumor motion in lung due to breathing and heartbeat, measured during radiotherapy. Int J Radiat Oncol Biol Phys 2002;53:822-34. 
[19] Knopf A, Nill S, Yohannes I, Graeff C, Dowdell S, Kurz C, et al. Challenges of radiotherapy: report on the 4D treatment planning workshop 2013. Phys Med 2014;30:809-15.

[20] Berbeco RI, Nishioka S, Shirato H, Jiang SB. Residual motion of lung tumors in end-of-inhale respiratory gated radiotherapy based on external surrogates. Med Phys 2006;33:4149-56.

[21] Onodera Y, Nishioka N, Yasuda K, Fujima N, Torres M, Kamishima T, et al. Relationship between diseased lung tissues on computed tomography and motion of fiducial marker near lung cancer. Int J Radiat Oncol Biol Phys 2011;79:1408-13.

[22] Poulsen PR, Cho B, Ruan D, Sawant A, Keall PJ. Dynamic multileaf collimator tracking of respiratory target motion based on a single kilovoltage imager during arc radiotherapy. Int J Radiat Oncol Biol Phys 2010;77:600-7.

[23] Kamino Y, Takayama K, Kokubo M, Narita Y, Hirai E, Kawawda N, et al. Development of a four-dimensional image-guided radiotherapy system with a gimbaled X-ray head. Int J Radiat Oncol Biol Phys 2006;66:271-8.

[24] Hoogeman M, Prevost JB, Nuyttens J, Poll J, Levendag P, Heijmen B. Clinical accuracy of the respiratory tumor tracking system of the cyberknife: assessment by analysis of log files. Int J Radiat Oncol Biol Phys 2009;74:297-303.

[25] Katoh N, Onimaru R, Sakuhara Y, Abo D, Shimizu S, Taguchi H, et al. Real-time tumor-tracking radiotherapy for adrenal tumors. Radiother Oncol 2008;87:41824.

[26] Imura M, Yamazaki K, Shirato H, Onimaru R, Fujino M, Shimizu S, et al. Insertion and fixation of fiducial markers for setup and tracking of lung tumors in radiotherapy. Int J Radiat Oncol Biol Phys 2005;63:1442-7.

[27] Imura M, Yamazaki K, Kubota KC, Itoh T, Onimaru R, Cho Y, et al. Histopathologic consideration of fiducial gold markers inserted for real-time tumor-tracking radiotherapy against lung cancer. Int J Radiat Oncol Biol Phys 2008;70: $382-4$.

[28] Jang SS, Huh GJ, Park SY, Yang PS, Cho EY. The impact of respiratory gating on lung dosimetry in stereotactic body radiotherapy for lung cancer. Phys Med 2014;30:682-9.

[29] Ueki N, Matsuo Y, Nakamura M, Mukumoto N, Iizuka Y, Miyabe Y, et al. Intraand interfractional variations ingeometric arrangement between lung tumours and implanted markers. Radiother Oncol 2014;110:523-8. 